\title{
Development of an oral cancer recurrence mouse model after surgical resection
}

\author{
ANDREAS BEHREN $^{1,6}$, YORK KAMENISCH ${ }^{2}$, SABRINA MUEHLEN ${ }^{1}$, CHRISTA FLECHTENMACHER ${ }^{3}$, \\ UWE HABERKORN $^{4}$, HERMANN HILBER ${ }^{2}$, JEFFREY N. MYERS ${ }^{5}$, ZAZIE BERGMANN ${ }^{1}$, \\ PETER KARL PLINKERT $^{1}$ and CHRISTIAN SIMON ${ }^{1}$
}

\begin{abstract}
${ }^{1}$ Department of Otolaryngology, Head and Neck Surgery, University Hospital of Heidelberg, 69120 Heidelberg; ${ }^{2}$ Department of Otolaryngology, Head and Neck Surgery, University Hospital of Tuebingen, 72076 Tuebingen; ${ }^{3}$ Department of Pathology, ${ }^{4}$ Department of Nuclear Medicine, Department of Radiology, University Hospital Heidelberg, 69120 Heidelberg, Germany;

${ }^{5}$ Department of Head and Neck Surgery, University of Texas, M.D. Anderson Cancer Center, Houston, TX 77098, USA
\end{abstract}

Received September 17, 2009; Accepted October 27, 2009

DOI: 10.3892/ijo_00000562

\begin{abstract}
Oral squamous cell carcinomas (OSCC) are frequent epithelial malignancies and afflicted with a poor prognosis. The majority of these cancers are treated with surgical resection and local recurrences are predominantly responsible for a fatal outcome. In order to provide a better understanding of the development of these local recurrences after surgical ablation, we developed an orthotopic floor-ofmouth squamous cell carcinoma murine model, in which local recurrences occur at a high frequency $(55 \%, 8$ out of 15 mice) within 6-21 days after microsurgical removal of the primary. Expression of the enhanced green fluorescent protein (eGFP) in the cancer cells allows in this new model to confirm complete surgical resection under the microscope and helps to track repopulating primary tumor cells in the local recurrence by optical imaging. In addition the model resembles all typical features of invasive head and neck cancers including the formation of lymph node metastasis and local infiltration.
\end{abstract}

Correspondence to: Dr Christian Simon, HNO-Universitätsklinik Heidelberg, Im Neuenheimer Feld 400, 69120 Heidelberg, Germany

E-mail: csimon1407@aol.com or christian.simon@med.uniheidelberg.de

Present address: ${ }^{6}$ Ludwig Institute for Cancer Research Ltd., Australia

Abbreviations: eGFP, enhanced green fluorescent protein; OSCC, oral squamous cell carcinoma; HNSCC, head and neck squamous cell carcinoma

Key words: head and neck squamous cell carcinoma, oral cancer, recurrence, mouse model, metastasis, enhanced green fluorescent protein

\section{Introduction}

Head and neck squamous cell cancer (HNSCC) is the 6th most common cancer in humans and afflicted with a poor prognosis. HNSCCs, that include cancers of the oral cavity (OSCC), paranasal sinuses, pharynx and larynx, account for more than 615,000 cases per year worldwide and for more than 200,000 deaths (1). The most commonly involved site is the oral cavity with squamous cell carcinomas representing $90 \%$ of these cancers (2).

The overall 5-year survival rate in patients suffering from OSCC has remained around $50 \%$ for several decades. Surgical resection is the mainstay of the treatment of these cancers with or without adjuvant radiation therapy. Despite surgical advances in the field the formation of local recurrences remains the major plague of this disease and is correlated with poor prognosis (1). Thus, an animal model to investigate the development of such recurrences after surgery is required that will aid in the understanding of underlying biological mechanisms and the consecutive design of new treatment strategies.

Local tumor recurrences consist of host (i.e. fibroblasts, endothelial cells, macrophages, mast cells) and primary regrowing tumor cells (3). The latter cell population is believed to contain tumor stem cells that significantly contribute to the establishment of recurrent disease (3). An animal model that is supposed to aid in the identification and elucidation of biological mechanisms that are underlying the process of local recurrence formation has to allow to separately study these cell populations, particularly the re-growing primary tumor cells.

Labeling of cancer cells via the stable expression of bioluminescent or fluorescent markers such as GFP from the jellyfish Aquorea victoria has been used in various animal models (4-16). Besides the advantage of visualizing tumor cells directly and identifying neo-angiogenesis of tumorbearing sites $(17,18)$, GFP has been successfully used for the detection of distant metastases in a large number of animal studies $(9,11,15,19-22)$. Moreover, GFP has been used in an orthotopic tongue cancer model to visualize lymph node metastases (23). 
In the present study we injected a stable eGFP-expressing clone from a murine squamous cell carcinoma cell line (SCC7) into the floor-of-mouth region of athymic BALB/ c-NU mice. A trans-cervical route of tumor cell injection was purposely chosen to create tumors growing in the submental region of the jaw as opposed to the oral cavity. This was done in order to create tumors more easily accessible for surgical removal and to avoid complications that would be inevitable, if a trans-oral resection was attempted. Fluorescence microscopy and real-time photon imaging was used to control for complete microsurgical removal of the tumor and identification of eGFP positive tumor cells within the recurrent local tumors.

In summary, our data indicate that we have created a reliable and reproducible mouse model for local recurrences of oral cavity cancers. The use of eGFP-expressing clones derived from epithelial cancer cell lines serves within this model as a means to confirm complete tumor resection and may help to track repopulating primary tumor cells within the recurrent disease.

\section{Materials and methods}

Cell lines. SCC7 cells were a gift from Lukas Mach (University of Vienna) and were cultured in MEM $\alpha$ medium (PAA, Linz, Austria) supplemented with $10 \%$ FBS. SCC7 is a murine squamous cell carcinoma cell line that spontaneously arose in $\mathrm{C} 3 \mathrm{H} / \mathrm{Ej}$ mouse. eGFP-expressing clones were grown in the same medium containing $500 \mu \mathrm{g} / \mathrm{ml} \mathrm{G} 418$ for selection of stable transfected clones.

Generation of stable eGFP-expressing cell lines. SCC7 cells were plated out in $100-\mathrm{mm}^{2}$ culture dishes and transfected at $80 \%$ confluency with $2 \mu \mathrm{g}$ of an eGFP-encoding plasmid (pcDNA3-eGFP) using Lipofectamine ${ }^{\mathrm{TM}}$ (Invitrogen, Karlsruhe, Germany). The plasmid was obtained from U. Lauer (Medical Clinic, University of Tuebingen, Germany). Twenty-four hours after transfection cells were cultured in the presence of G418 (500 $\mu \mathrm{g} / \mathrm{ml})$ for several days. Stable transfected cells were picked and fluorescence detected by a fluorescence microscope (MZ FL-III; Leica, Solms, Germany).

Animal model. The experimental protocol was approved by the 'Regierungspraesidium Tuebingen' and 'Karlsruhe' and accepted under HN 3/01 and 35-9185.81/G-21/07. Cells $\left(2 \times 10^{6}\right)$ suspended in $100 \mu 11 / 5$ Matrigel/HBSS solution (clonal growth experiments) or $8 \times 10^{5}$ cells in $50 \mu 11 / 3$ Matrigel/HBSS solution (recurrence experiments) were injected trans-cervically into the floor-of-mouth region of mice anesthetized with isofluran. Mice were sacrificed using $\mathrm{CO}_{2}$ or went on to surgery when the primary tumor size reached $0.8 \mathrm{~cm}$ in any dimension.

Anesthesia of the 6-week old female BALB/c-NU mice (Charles River, Bad Sulzfeld, Germany) prior to the surgery was performed via intraperitoneal injection of Rompun/ Xylazin $(10 \mathrm{mg} / \mathrm{kg})$ and Ketanest $(90 \mathrm{mg} / \mathrm{kg})$ or Domitor/ Midazolam/Fentanyl $(0.175 \mathrm{ml} / 25 \mathrm{~g})$. Anesthesia was antagonized as needed using Antisedan/Anexate/Naloxon $(0.21 \mathrm{ml} / 25 \mathrm{~g})$ once the skin incision was closed. Tumor size and weight of mice were measured every two days prior and after the surgery, tumor volume was calculated using the formula for ellipsoid bodies.

Surgery was carried out when the tumor reached a size of approximately $0.8 \mathrm{~cm}$ in any dimension. Mice were followed up for 8 weeks and animals developing recurrent disease were sacrificed once the tumor size reached $1 \mathrm{~cm}$. The mice with recurrent tumors and the recurrences were then made subject to real-time photon imaging analysis.

Surgery was performed using a binocular microscope with 5-fold magnification. The mouse was prepped using $70 \%$ ethanol and immobilized onto a preheated $\left(37^{\circ} \mathrm{C}\right)$ platform. A horizontal incision through skin and subcutaneous tissue was carried out directly above the tumor and flaps elevated superiorly and inferiorly. Once the entire tumor and main neck vessels and trachea were exposed, the tumor was excised using micro-scissors and micro-bipolar forceps for hemostasis avoiding any injury to these structures. Care was taken to allow for an adequately wide safety margin of healthy tissue around the tumor and complete resection was confirmed via fluorescent light and real-time photon imaging. Once the tumor was excised the wound was closed with a 7.0 nylon suture. Treatment of peri-operative pain was performed according to protocol with buprenorphin $100 \mu \mathrm{g}$ s.c. q $12 \mathrm{~h}$ for 2 days.

Detection of tumor tissue fluorescence in vivo and during surgery. Identification of fluorescent lymph nodes was performed after raising a skin flap over the neck fat pad and primary tumor during the surgical procedure under fluorescent light. Identification of fluorescent primary tumors and recurrences was performed using either a real-time photonimager system (Photon Imager; Biospace, Paris, France) or under fluorescent light. Emitted photons were counted over a time of $10 \mathrm{~min}$, acquired images were converted to pseudocolor, with bioluminescence signal shown in red and the weakest in blue.

Histology. At the time of necropsy the neck fat pad including the regional lymph nodes and the floor of the mouth including the tongue but without the mandible were fixed in $4 \%$ buffered formaldehyde. The specimens were then embedded in paraffin, sectioned, stained with hematoxylin and eosin and examined under the microscope. Histological studies on the primary tumors and lymph nodes were performed at the Institute for Pathology at the University Hospital Heidelberg and the Institute for Pathology at the University of Tuebingen.

\section{Results}

Tumor take, growth and in vivo visualization of different eGFP clones injected into the floor-of-mouth of BALB/c-NU mice. We established several eGFP-expressing cell clones derived from a mouse squamous cell carcinoma cell line (SCC7). EGFP expression was confirmed for at least 8 weeks by fluorescence microscopy (Fig. 1A shows the distribution of eGFP expression in clone 2). Generating stable clones often results in phenotypic changes. As a consequence, we examined stable eGFP-expressing clones for their in vivo growth characteristics and fluorescence. 
A

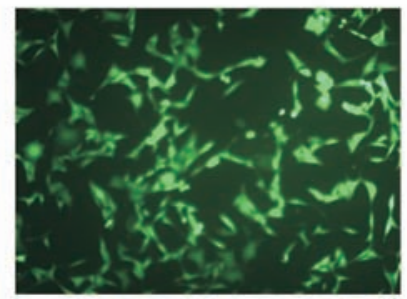

B
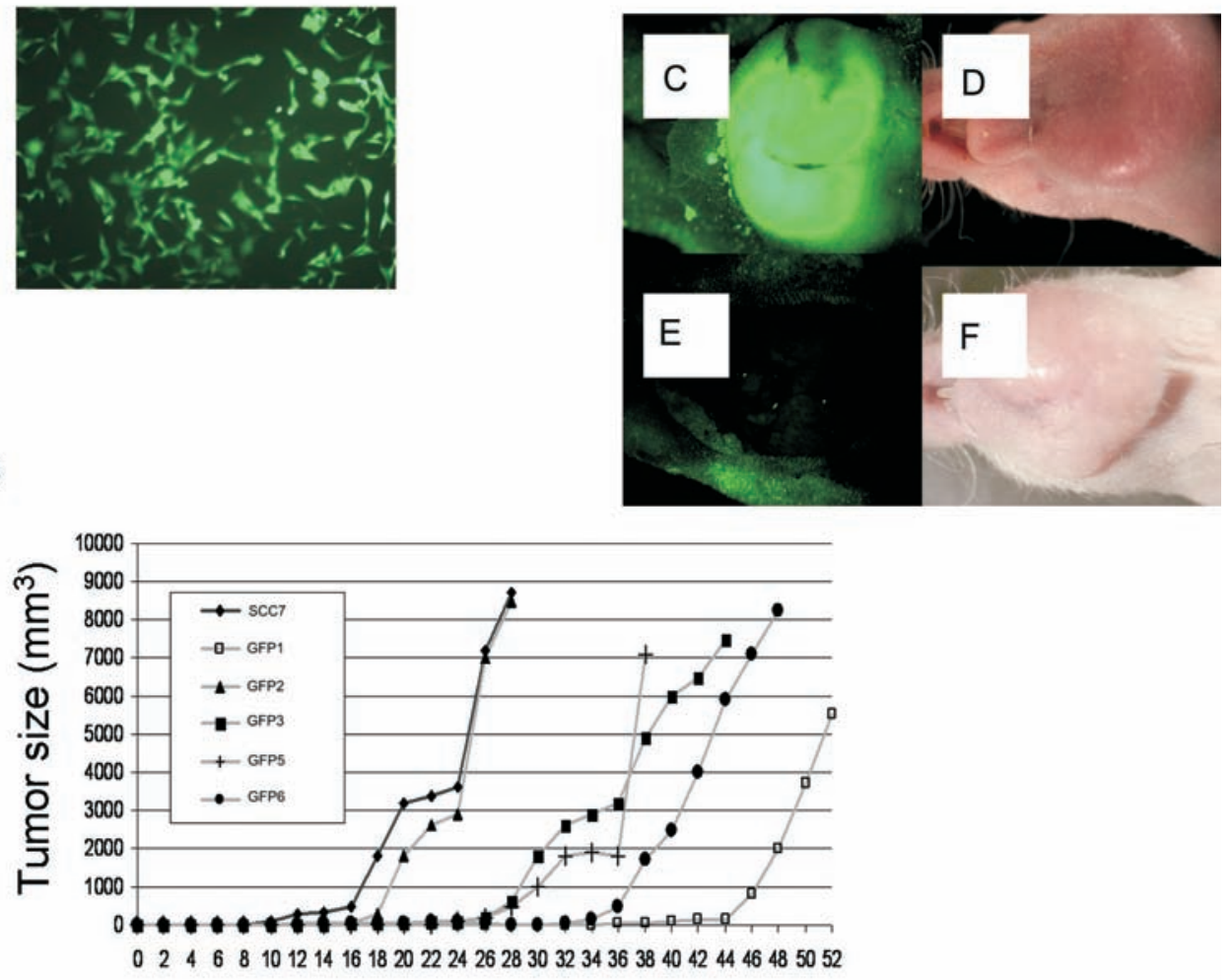

Days after cell implantation

Figure 1. The eGFP-positive SCC7 clone 2 forms fluorescent tumors in the floor-of-mouth region of BALB/c-NU mice and displays similar growth characteristics as the maternal cell line. SCC7 cells were stably transfected with pCDNA3-eGFP using Lipofectamine. Stable eGFP expression was confirmed over 8 weeks via serial observations measuring fluorescence. The picture shows eGFP-expressing cells (clone 2) using x40 magnification (A). After injection of eGFP-positive clones, tumor take and growth were assessed every other day. Length, width and height of the primary tumor were measured with a caliper. The volume of the tumor was calculated according to the formula of ellipsoid bodies. Mice were sacrificed or made subject to further experiments once tumors reached $8000 \mathrm{~mm}^{3}$. Tumor size was plotted against time; data represent one of each primary tumor (B). Fluorescence of primary tumors derived from clone 2 cells was assessed in living animals after raising a skin flap (C and D) and compared with tumors derived from GFP-negative maternal SCC7 cells (E and F). Pictures were taken using a Leica DCF300 camera.

Table I. Tumor growth characteristics in BALB/c-NU mice. ${ }^{\mathrm{a}}$

Cell line $2 \times 10^{6}$ cells Tumor outcome after injection Time-point of maximal volume Mice with tumor/injected mice

\begin{tabular}{lllr}
\hline SCC7 & Day10/Day 12 & Day 28/Day 33 & $2 / 2$ \\
SCC7 clone 1 & Day 36 & Day 51 & $1 / 2$ \\
SCC7 clone 2 & Day 12/Day 15 & Day 33/Day 27 & $2 / 2$ \\
SCC7 clone 3 & Day 12 & Day 44 & $1 / 2$ \\
SCC7 clone 4 & No & No & $0 / 2$ \\
SCC7 clone 5 & Day 24 & Day 40 & $1 / 2$ \\
SCC7 clone 6 & Day 12 & Day 48 & $1 / 2$ \\
SCC7 clone 7 & No & No & $0 / 2$ \\
SCC7 clone 8 & No & No & $0 / 2$
\end{tabular}

${ }^{a}$ The data are derived from observations of two mice. The growth characteristics of clone 2 were similar to the parental cell line.

The onset of tumor growth was markedly variable with only clone 2 resembling the growth characteristics of the parental cell line (Fig. 1B). Overall, tumor growth in the floor-ofmouth region was found in 6 out of 16 injected mice. No tumor growth occurred upon injection of clone 4, 7 and 8, and was observed in one mouse each upon using clones 1, 3, 5 and 6 .
In contrast tumor establishment was observed in both mice once either the parental cell line or clones 2 were used (Table I).

Of note, none of the other cell clones developed tumors earlier than the parental cell line suggesting that the generation of stable eGFP transfectants creates rather less aggressive cell lines. 

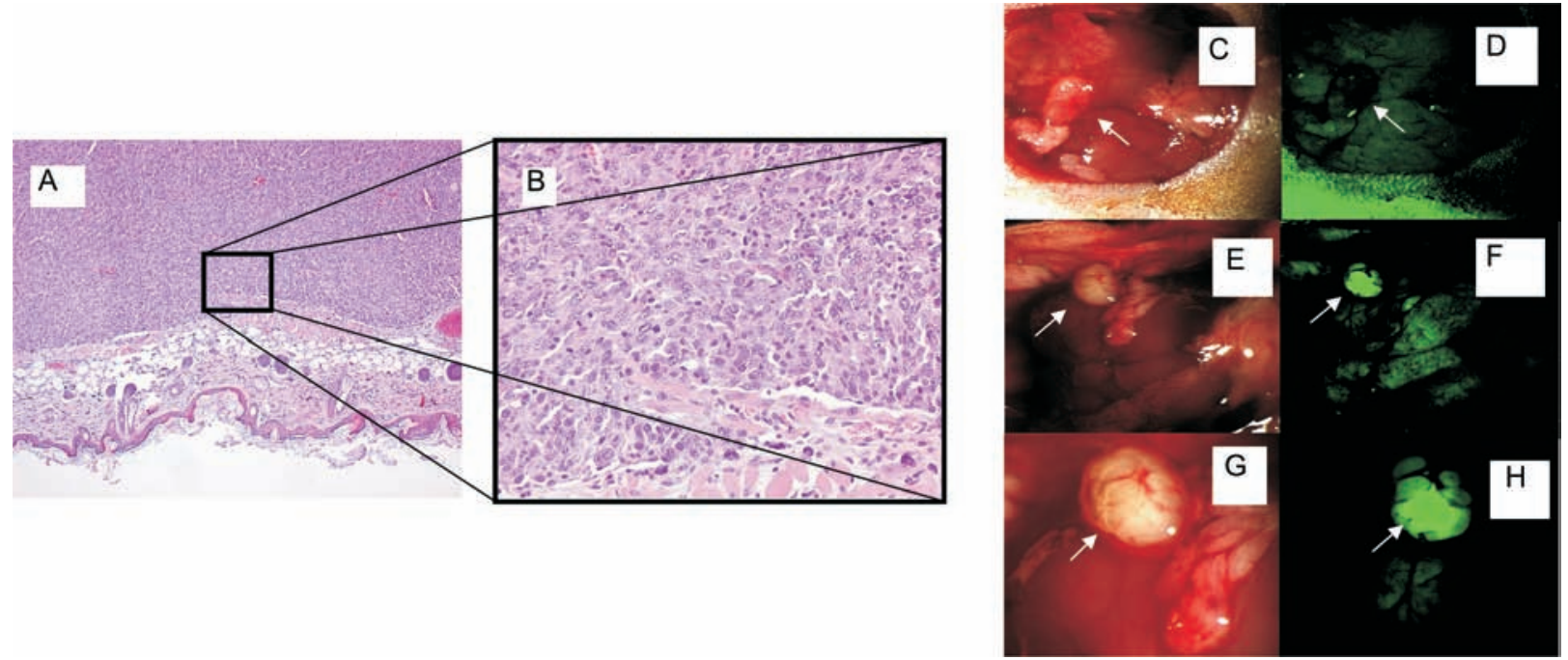

Figure 2. Tumors formed from the eGFP-expressing clone 2 are invasive and induce lymph node metastases. Histological examination of 5 tumors derived from clone 2 demonstrates local infiltration into surrounding muscle (A and B). In order to examine for the presence of cervical lymph node metastases, the neck fat pad of the animal was exposed and examined using regular (C, E and G) and UV light (D, F and H) conditions. Lymph node metastasis that was confirmed via conventional histology identified in mice implanted with maternal SCC7 tumor cells showed no fluorescence (C and D, white arrow), whereas mice implanted with eGFP-expressing cells developed lymph node metastases that were easily detected under UV light (D, F and $\mathrm{H}$ white arrow). G and $\mathrm{H}$ show magnifications from the lymph node in $\mathrm{E}$ and $\mathrm{F}$.
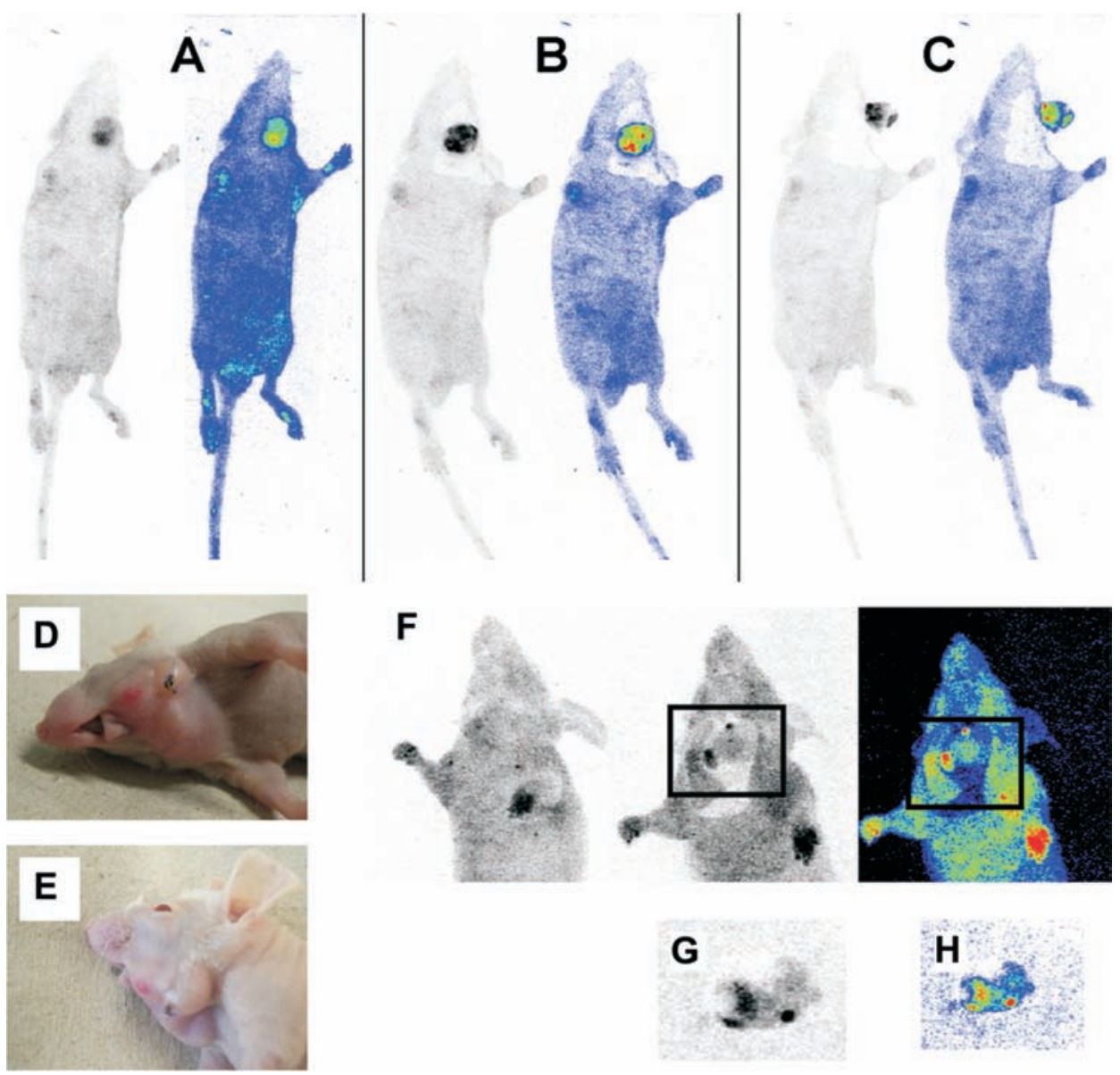

Figure 3. Complete microsurgical resection of tumors is confirmed via the absence of fluorescence and eGFP-positive areas can be identified in all recurrent tumors. Mice harboring clone 2-derived tumors were examined under normal and fluorescent light conditions in a two-photon counter chamber equipped with an eGFP filter set after surgery. Displayed are colored and grey-scale pictures of the same animal in (A) presurgical condition, (B) lifted skin flap, and (C) after tumor removal. After surgical removal of the primary tumor (tumor size of $\sim 0.8 \mathrm{~cm}$ in any dimension) and adequate wound healing, recurrences were found within 6-21 days. These progressed into a tumor measuring up to $1 \mathrm{~cm}$ in any dimension within the following 7-21 days. Mice were sacrificed thereafter (D and $\mathrm{E}$ ) and fluorescence determined using the two-photon counter $(\mathrm{F}, \mathrm{G}$ and $\mathrm{H})$. 
Table II. Recurrent disease in animals injected with clone 2.

\begin{tabular}{|c|c|c|c|c|}
\hline Injected cells $\left(8 \times 10^{5}\right)$ & Tumor onset & Injected mice/tumors & Recurrence onset after $\mathrm{OP}^{\mathrm{a}}$ & Operated mice/recurrences ${ }^{b}$ \\
\hline SCC7 clone 2 & Day 12-36 & $20 / 16$ & Day 6-21 & $15 / 8$ \\
\hline
\end{tabular}

$0.6-0.8 \mathrm{~cm}$ in one dimension. ${ }^{\mathrm{b}}$ One out of 16 mice with tumor died of an unknown reason.

Secondly, we investigated, if tumors derived from an eGFP-positive clone that exhibits similar growth characteristics compared with the maternal cell line (clone 2) exhibits fluorescence in vivo. Thus, after trans-cervical injection into the submental region of BALB/c-NU mice and growth for 28 days the two established tumors were examined in the living mice under fluorescent light after raising a skin flap and a strong fluorescence was observed in both animals (Fig. 1C and D). Tumors derived from the parental cell line SCC7 (Fig. 1E and F) showed no fluorescent signal.

Our experiments so far suggest that clone 2 combines the features of stable eGFP expression with maintenance of in vivo growth characteristics. We therefore used this clone for all further experiments.

Injection of clone 2 into the floor-of-mouth of $B A L B / c-N U$ mice results in the formation of invasive tumors that metastasize to regional lymph nodes. In order to investigate if clone 2 and SCC7 cells both possess a similar capacity to form tumors in nude mice, we injected SCC7 and clone 2 cells, respectively, into 20 mice each. In both groups 18 mice developed tumors in a similar time period after the injection; 6-12 days (SCC7) and 7-14 days (clone 2).

Invasive growth is a typical feature of cancers of the head and neck (24) and therefore we examined 5 primary tumors derived from the injection of clone 2 for histological evidence of local invasion and found infiltration of surrounding musculature in all specimens (Fig. 2A and B).

The development of lymph node metastases is typical for head and neck cancers (2). To investigate, if clone 2 -derived primary tumors give rise to the development of regional metastases, we examined 2 mice injected with SCC7 cells and 2 mice injected with clone 2 for the presence of lymph node metastases. This was done through the examination of the neck fat pad under fluorescent light, which was followed by embedding and histological examination after HE-staining (data not shown). With this approach we could identify lymph node metastases in both mice carrying tumors from the parental cell line SCC7 (Fig. 2C and D) and in one out of two tumor-bearing animals injected with clone 2. The regional metastases in the animal injected with clone 2 could be easily detected via eGFP-dependent green-fluorescence after raising a skin flap exposing the soft tissue of the neck (Fig. 2E-H). This suggests the capacity of clone 2 to generate regional metastases similar to the maternal cell line SCC7 with the additional feature of bioluminescence of the metastatic cells.

Development of local recurrences that maintain localized eGFP expression after microsurgical removal of the primary.
The two goals of this study are the development of a localrecurrence model for oral cavity cancers after surgical removal and to answer the question if it is feasible to detect repopulating tumor cells originating from the primary tumor in the recurrence via eGFP. Considering the major advances in the field of microsurgery and the use of the operative microscope, we hypothesized that it is feasible to perform major resections of tumors growing in the floor-of-mouth region up to a size of $0.8 \mathrm{~cm}$ in any diameter without creating major peri-operative morbidity. Thus, we injected 20 mice with $8 \times 10^{5}$ SCC7 clone 2 cells into the floor-of-mouth. A lower cell number was used in this experiment to allow for a longer growth period and thus more extensive local invasion prior to the surgical procedure. We were able to induce primary tumor growth in 16 out of 20 mice within 12-36 days and microsurgically removed the tumors in 15 of them. One mouse died in the peri-operative period. We controlled for complete microsurgical removal of the primary cancer via fluorescence and real-time photon imaging (Fig. 3A-C) and detected recurrent tumors in 8 out of 14 mice within 6-21 days (Fig. 3D and E) (Table II). The overall surveillance period after surgery was 6 weeks. The level of fluorescence in the recurrences was found to be lower as compared to the primary tumors and localized to only scattered areas (Fig. 3F-H). Our data indicate the suitability of this model to study the development of local recurrences after microsurgical removal. In addition this model provides an interesting tool to examine repopulating tumor cells in the local recurrence by means of eGFP tracking.

\section{Discussion}

The first orthotopic head and neck tumor model was reported in 1990 by Dinesman and colleagues (25). We have previously introduced an orthotopic floor-of-mouth cancer model using immuno-deficient mice with a human HNSCC cell line (UMSCC1) $(26,27)$. Herein, we demonstrated that tumors formed from this cell line readily infiltrate the different muscle layers of the floor-of-mouth in a step-wise fashion that is associated with vascular and bony infiltration, perineural invasion and formation of lymph node metastases (26). In the present study we are extending this model into a local oral cavity cancer-recurrence model after surgical therapy through the application of microsurgical techniques.

The formation of local recurrences is known to be the consequence of an interaction between repopulating primary tumor and host cells that are comprised of fibroblasts, endothelial and other immunogenic cells (3). The repopulating 
cells derived from the primary tumor are considered tumor stem cells and believed to play a key role in the development of local recurrences. Studies aiming towards the characterization of these repopulating tumor cells in animal models have been hampered by the investigators inability to differentiate between primary tumor cells that are growing back and cells derived from the host site. We have been able to detect eGFP fluorescence corresponding to eGFP-positive primary tumor cells in distinct areas of all recurrences. This suggests that by using this model it may become possible to differentiate between cellular sub-populations within the recurrence, which may aid in the identification and further characterization of cells with stem cell characteristics.

Local recurrences were found in 8 out of 14 mice $(55 \%)$ that underwent surgery. The rate of recurrence is certainly biased by the technique and the surgeon. If these procedures are performed in the operating theatre, frozen sections are taken from the patient to confirm complete and thus adequate resection. However this poses a problem in nude mice, since in the floor-of-mouth region of these animals is simply not enough tissue available for this kind of analysis. Thus, our solution to this problem was to confirm complete resection via the absence of fluorescent signatures under the microscope.

Surgical procedures on mice may appear technically demanding due to the small size of the animals, tumors and delicacy of anatomical neck structures. However, employing microsurgical techniques that include the use of a surgical microscope and instruments generally used for microvascular surgery allows for surgical removal of complex tumors such as those located in the head and neck region while the surgery related morbidity remains low.

Initially we attempted to develop a syngeneic, immunecompetent murine cancer model. However, within the preliminary experiments with immune-competent $\mathrm{C} 3 \mathrm{H} / \mathrm{HeJ}$ mice, we noted a very poor tumor-take rate of our clones, slow growth, and even regression in one animal. We subjected spleen cells from such animals to lymphocyte transformation tests and found a significant proliferation only after challenge with cell lysates from eGFP clones harvested from mice that failed to develop tumors or showed tumor regression suggesting an immune response against eGFP. We therefore chose BALB/c-NU mice to overcome eGFP induced immune reactions.

Another quite well-established model for head and neck squamous cell carcinomas is the VX2 carcinoma model in New Zealand white rabbits (28). Tumor formation takes place rather rapidly and metastatic disease to lymph nodes occurs at a frequency comparable to the human system (28). Even local resections have been performed in this model and recurrence formation was observed in $7 \%$ of the animals (29). Although these data somewhat mirror the current treatment outcome in humans there are drawbacks to the model. Working in rabbits is more difficult, because animal housing is limited and high animal numbers for statistical analyses come at unreasonable costs. The duration of onset of local recurrences is certainly closer to human reality but lacks versatility due to length of duration. Finally, the low number of recurrences demands high numbers of animals in order to generate reliable data.
In conclusion this model may provide a means to elucidate biological mechanisms leading to cancer recurrence formation after surgery of oral cavity cancers.

\section{Acknowledgement}

We are grateful to Lukas Mach (University of Vienna) for the SCC7 cells and to Ulrich Lauer (Medical Clinic, University of Tuebingen, Germany) for the pcDNA3-eGFP construct. We would like to especially thank Marlies Knipper and Goran Vucelic (University Hospital Tuebingen) for extensive support during the experimental part in Tuebingen and Karin Leotta and Hadeel Khallouf (University Hospital Heidelberg) for support in Heidelberg. This work was supported by the Fortune program of the University of Tuebingen (889-1-0), a DFG grant (Si 634-4/1, Si 634-5/1), and a Deutsche Krebshilfe e.V. grant (107689) to C. Simon.

\section{References}

1. Stewart BWKP (ed): World Cancer Report. IARC-Press, Lyon, 2003.

2. Dobrossy L: Epidemiology of head and neck cancer: magnitude of the problem. Cancer Metastasis Rev 1: 9-17, 2005.

3. Bissell MJ, Labarge MA: Context, tissue plasticity, and cancer: are tumor stem cells also regulated by the microenvironment? Cancer Cell 1: 17-23, 2005.

4. Liu T, Ding Y, Xie W, Li Z, Bai X, Li X, Fang W, Ren C, Wang S, Hoffman RM and Yao K: An imageable metastatic treatment model of nasopharyngeal carcinoma. Clin Cancer Res 13: 3960-3967, 2007

5. Kishimoto H, Kojima T, Watanabe Y, Kagawa S, Fujiwara T, Uno F, Teraishi F, Kyo S, Mizuguchi H, Hashimoto Y, Urata Y, Tanaka $\mathrm{N}$ and Fujiwara T: In vivo imaging of lymph node metastasis with telomerase-specific replication-selective adenovirus. Nat Med 10: 1213-1219, 2006.

6. Moshitch-Moshkovitz S, Tsarfaty G, Kaufman DW, Stein GY, Shichrur K, Solomon E, Sigler RH, Resau JH, Vande Woude GY and Tsarfaty I: In vivo direct molecular imaging of early tumorigenesis and malignant progression induced by transgenic expression of GFP-Met. Neoplasia 8: 353-363, 2006.

7. Bouvet M, Wang J, Nardin SR, Nassirpour R, Yang M, Baranov E, Jiang P, Moossa AR and Hoffman RM: Real-time optical imaging of primary tumor growth and multiple metastatic events in a pancreatic cancer orthotopic model. Cancer Res 5: 1534-1540, 2002.

8. Hasegawa S, Yang M, Chishima T, Miyagi Y, Shimada H, Moossa AR and Hoffman RM: In vivo tumor delivery of the green fluorescent protein gene to report future occurrence of metastasis. Cancer Gene Ther 7: 1336-1340, 2000.

9. Kaneko K, Yano M, Tsujinaka T, Morita S, Taniguchi M, Fujiwara Y, Doki Y, Inoue M, Shiozaki H and Monden M: Establishment of a visible peritoneal micrometastatic model from a gastric adenocarcinoma cell line by green fluorescent protein. Int J Oncol 16: 893-898, 2000.

10. Paris S, Chauzy C, Martin-Vandelet N, Delpech B, Thiberville L, Martin JP and Diarra-Mehrpour M: A model of spontaneous lung metastases visualised in fresh host tissue by green fluorescent protein expression. Clin Exp Metastasis 10: 817-822, 1999.

11. Yang M, Jiang P, Sun FX, Hasegawa S, Baranov E, Chishima T, Shimada H, Moossa AR and Hoffman RM: A fluorescent orthotopic bone metastasis model of human prostate cancer. Cancer Res 4: 781-786, 1999.

12. Stripecke R, Carmen Villacres M, Skelton D, Satake N, Halene S and Kohn D: Immune response to green fluorescent protein: implications for gene therapy. Gene Ther 7: 1305-1312, 1999.

13. Hoffman RM: Orthotopic transplant mouse models with green fluorescent protein-expressing cancer cells to visualize metastasis and angiogenesis. Cancer Metastasis Rev 3: 271-277, 1998.

14. Moore A, Sergeyev N, Bredow S and Weissleder R: A model system to quantitate tumor burden in locoregional lymph nodes during cancer spread. Invasion Metastasis 4: 192-197, 1998. 
15. Yang $M$, Hasegawa $S$, Jiang $P$, Wang $X$, Tan $Y$, Chishima $T$, Shimada H, Moossa AR and Hoffman RM: Widespread skeletal metastatic potential of human lung cancer revealed by green fluorescent protein expression. Cancer Res 19: 4217-4221, 1998.

16. Chishima T, Yang M, Miyagi Y, Li L, Tan Y, Baranov E, Shimada H, Moossa AR, Penman S and Hoffman RM: Governing step of metastasis visualized in vitro. Proc Natl Acad Sci USA 21: 11573-11576, 1997.

17. Hoffman RM: Imaging tumor angiogenesis with fluorescent proteins. APMIS 7-8: 441-449, 2004.

18. Hoffman R: Green fluorescent protein imaging of tumour growth, metastasis, and angiogenesis in mouse models. Lancet Oncol 9: 546-556, 2002.

19. Chishima T, Miyagi Y, Wang X, Yamaoka H, Shimada H, Moossa AR and Hoffman RM: Cancer invasion and micrometastasis visualized in live tissue by green fluorescent protein expression. Cancer Res 10: 2042-2047, 1997.

20. Chishima T, Miyagi Y, Wang X, Baranov E, Tan Y, Shimada H, Moossa AR and Hoffman RM: Metastatic patterns of lung cancer visualized live and in process by green fluorescence protein expression. Clin Exp Metastasis 5: 547-552, 1997.

21. Yang M, Baranov E, Jiang P, Sun FX, Li XM, Li L, Hasegawa S, Bouvet M, Al-Tuwaijri M, Chishima T, Shimada H, Moossa AR, Penman S and Hoffman RM: Whole-body optical imaging of green fluorescent protein expressing tumors and metastases. Proc Natl Acad Sci USA 97: 1206-1211, 2000.

22. MacDonald TJ, Tabrizi P, Shimada H, Zlokovic BV and Laug WE: Detection of brain tumor invasion and micrometastasis in vivo by expression of enhanced green fluorescent protein. Neurosurgery 6: 1437-1442, 1998.
23. Myers JN, Holsinger FC, Jasser SA, Bekele BN and Fidler IJ: An orthotopic nude mouse model of oral tongue squamous cell carcinoma. Clin Cancer Res 1: 293-298, 2002.

24. Kademani D, Bell RB, Bagheri S, Holmgren E, Dierks E, Potter B and Homer L: Prognostic factors in intraoral squamous cell carcinoma: the influence of histologic grade. J Oral Maxillofac Surg 11: 1599-1605, 2005.

25. Dinesman A, Haughey B, Gates G and Aufdemorte T: Development of a new in vivo model for head and neck cancer. Laryngoscope 766-774, 1990.

26. Simon C, Nemechek AJ, Boyd D, O'Malley BW Jr, Goepfert H, Flaitz CM and Hicks MJ: An orthotopic floor-of-mouth cancer model allows quantification of tumor invasion. Laryngoscope 11: 1686-1691, 1998

27. Simon C, Hicks MJ, Nemechek AJ, Mehta R, O'Malley BW Jr, Goepfert H, Flaitz CM and Boyd D: PD 098059, an inhibitor of ERK1 activation, attenuates the in vivo invasiveness of head and neck squamous cell carcinoma. Br J Cancer 9: 1412-1419, 1999.

28. Dunne AA, Schmidt A, Kuropkat C, Ramaswamy A, Schulz S and Werner JA: The auricular VX2 carcinoma - an animal model for sentinel node concept. In Vivo 5: 457-461, 2003.

29. Sapundzhiev N, Dunne AA, Ramaswamy A and Werner JA: The auricular VX2 carcinoma: feasibility of complete tumor resection. Anticancer Res 6B: 4209-4214, 2005. 